TITLE:

\title{
Model of insertion and release of a large solute into and from a biopolymer complex
}

$\operatorname{AUTHOR}(S)$ :

Amano, Ken-ich; Kinoshita, Masahiro

\section{CITATION:}

Amano, Ken-ich ... [et al]. Model of insertion and release of a large solute into and from a biopolymer complex. Chemical Physics Letters 2011, 504(4-6): 221-224

ISSUE DATE:

2011-03

URL:

http://hdl.handle.net/2433/157330

\section{RIGHT:}

@ 2011 Elsevier B.V.; この論文は出版社版でありません。引用の際には 出版社版をご確認ご利用ください。; This is not the published version. Please cite only the published version. 


\title{
Model of insertion and release of a large solute into and from a biopolymer complex
}

\author{
Ken-ich Amano ${ }^{1}$ and Masahiro Kinoshita ${ }^{2}$ \\ ${ }^{1}$ Graduate School of Energy Science, Kyoto University, Uji, Kyoto 611-0011, Japan \\ ${ }^{2}$ Institute of Advanced Energy, Kyoto University, Uji, Kyoto 611-0011, Japan
}

Author to whom correspondence should be addressed: Masahiro Kinoshita.

E-mail address: kinoshit@iae.kyoto-u.ac.jp.

\begin{abstract}
Insertion of a large solute into a biopolymer complex followed by release of the same solute from it is a principal function for sustaining life. We show that the switch from insertion to release is achieved by altering the solute conformation to reduce the excluded volume (EV) generated by the solute for solvent molecules and to increase the solute solvophilicity. The reduction in the EV weakens the insertion power induced by the entropic force, and the increase in the solvophilicity promotes preferential solvation not in the solvent confined within the complex cavity but in the bulk solvent.
\end{abstract}




\section{Introduction}

Insertion of a large solute into an even larger vessel comprising biopolymers followed by release of the same solute from it is a fundamental function in biological systems. We consider two typical examples: (I) An antibiotic molecule is inserted into the ATP-binding cassette (ABC) transporter from the inside of cell membrane, and then the molecule is released from the transporter to the outside (thus, diverse substrates are carried across the membrane) [1,2]; and (II) an unfolded protein is inserted into the chaperonin GroEL from bulk aqueous solution, protein folding occurs within the GroEL cavity, and the folded protein is released back to the bulk solution (a variety of proteins are inserted and released) [3,4]. It is mysterious that the two apparently opposite processes, insertion and release, successively occur in the same system. To the best of our knowledge, no theoretical works have tackled the question of the insertion/release function, and very little is known on its mechanism. By computer simulations, dynamics of structural changes of the transporter itself [5] and characteristics of protein folding within the GroEL cavity [6,7] have been studied, but neither insertion nor release of a solute has been treated. It seems that computer simulations are not capable of covering the time length required to demonstrate the insertion or release process.

There can be two major factors to be explored: (A) The switch from insertion to release is achieved by modifying geometric features and inner-surface properties of the vessel; and (B) it is achieved by altering the conformation of the solute. Though factor (A) is found in both of the two examples described above, it is particularly important in example (I) where the solute properties remain almost unchanged. The transporter takes the inward-facing structure for insertion while it takes the outward-facing structure for release, and the two structures are quite different. Factor (B) should be essential in example (II). In our view, solvation properties of the solute are changed by the alteration of its conformation. A protein becomes much more compact upon folding. Further, the exposed surface of an unfolded protein comprises solvophobic groups as well as solvophilic groups, but the protein becomes dominantly solvophilic after the folding is finished because solvophobic groups are preferentially buried. This study is focused on factor (B) in example (II).

It has been claimed that the entropic force, which originates from the translational displacement of solvent molecules, is a primary driving force in a variety of biological processes [8-16]. As a new aspect, we have recently suggested that the entropic force 
plays essential roles in the solute insertion into a vessel [15]. On the other hand, the release process has never been considered. The question is the following: How can the solute, which has already been inserted into the vessel and is constrained inside it, be released from it to the outside? Both of insertion and release must be explained consistently within the same theoretical framework. The general thought is that the underlying mechanism can be elucidated only if details of the polyatomic structures of the protein and biopolymer complex are taken into account. This makes matters quite complicated and may be a reason why no theoretical works have been reported.

In this study, we show that the main physics can be understood through a much simpler model: a model focused on solvation properties of a solute in the solvent confined on the scale of a nanometer which are substantially different from those in the bulk solvent. We analyze the potential of mean force (PMF) between a large spherical solute and an even larger vessel with cylindrical shape, which are immersed in small spheres forming the solvent. The analysis is made using the three-dimensional (3D) version $[8,10,11,15-17]$ of the integral equation theory, a statistical-mechanical theory for liquids. In one calculation, the $3 \mathrm{D}$ integral equation theory gives the spatial distribution of the PMF while a computer simulation gives only the value of the PMF on a single position. Further, in the theory the PMF can readily be decomposed into entropic and energetic components. We study effects of the magnitude of excluded volume (EV) generated by the solute and the strength of solute solvophobicity or solvophilicity on the two components. Here, the EV is the volume of the space which the centers of solvent molecules cannot enter. It is argued that the entropic component usually drives the insertion process while the energetic component can be requisite in the release process. The insertion/release function is exhibited through judicious adjustment of the two components.

\section{Model and Theory}

We wish to adopt the simplest possible model that still captures the essential physics. A large sphere with diameter $d_{\mathrm{B}}$ (solute 1) and a cylindrical vessel (solute 2), which are illustrated in Fig. 1, are immersed in the solvent at infinite dilution. The solvent is modeled as small spheres with diameter $d_{\mathrm{S}}$ and bulk density $\rho_{\mathrm{S}}$ interacting through the potential, 
$u_{\mathrm{SS}}(r)=\infty \quad$ for $r<d_{\mathrm{S}}$,

$u_{\mathrm{SS}}(r)=-\varepsilon\left(d_{\mathrm{S}} / r\right)^{6} \quad$ for $\quad r>d_{\mathrm{S}}$,

where $r$ is the distance between the centers of two small spheres. In potential (1), the repulsive part of the Lennard-Jones potential is simply replaced by a hard-core interaction. $\rho_{\mathrm{S}} d_{\mathrm{S}}{ }^{3}$ is set at the value for water under the normal condition, 0.7317 $\left(d_{\mathrm{S}}=0.28 \mathrm{~nm}\right)$, and $\varepsilon /\left(k_{\mathrm{B}} T\right)=1.0$ for $T=298 \mathrm{~K}$. The solute $I$-solvent $(I=1,2)$ interaction potential is taken to be

$\begin{array}{lll}u_{I \mathrm{~S}}(h)=\infty & \text { for } & h<d_{\mathrm{S}} / 2, \\ u_{I \mathrm{~S}}(h)=-\left(\xi_{I} / 8\right)\left(d_{\mathrm{S}} / h\right)^{3} & \text { for } & h>d_{\mathrm{S}} / 2,\end{array}$

where $h$ is the distance between the center of a small sphere and the nearest surface of solute $I$. In potential (2), whose physical pertinence was argued in our earlier publications $[18,19]$, the repulsive part of the 9-3 type potential is simply replaced by a hard-core interaction. To focus on effects of the properties of solute 1, geometric features of solute 2 and $\xi_{2}$ are all fixed $\left(\xi_{2} /\left(k_{\mathrm{B}} T\right)=1.5\right.$ for $\left.T=298 \mathrm{~K}\right)$. $\xi_{1}$ and $d_{\mathrm{B}}$ are varied as major parameters. The surface of solute 1 is solvophobic for small $\xi_{1}$ and solvophilic for large $\xi_{1}$.

Thanks to hydrogen bonds, water exists as a dense liquid despite the exceptionally small molecular size. However, the hydrogen bonds themselves are not crucial in reproducing many of the interesting characteristics of water. For example, as shown in our earlier work [20], the hydrophobicity can reasonably be elucidated by modeling water as spherical particles interacting through strongly attractive potential like that expressed by Eq. (2), as long as the particle size and number density are set at the values for water, respectively.

The details of the 3D integral equation theory were described in our earlier publications $[8,10,11,15-17]$. Here we mention how to specify potential (2) for solute 2 $(I=2)$. In this theory, the numerical values of the potential are calculated on 3D grid points. On a grid point, we determine the distance between the center of the small sphere placed on this grid point and the nearest surface of the vessel. The distance is then substituted into $h$ for calculating potential (2). With this simple treatment, a small sphere feels significant, negative potential only in the close vicinity of the surface not only of the side but also of the base of the vessel (the potential becomes stronger as the small sphere approaches the surface), and the cylindrical dependence of the potential is 
fully taken into account. This property is all we need for the spatial distribution of the solvent-solute 2 potential. Moreover, we have verified that the results obtained are considerably robust against the details of the calculation procedure for the spatial distribution.

Solute 1-solvent and solute 2-solvent correlation functions are first calculated from solvent-solvent correlation functions, and then the $\operatorname{PMF} \Phi(x, y, z)$ between solutes 1 and 2 are obtained. Its physical meaning [15,16] can be understood from

$\Phi(x, y, z)=F(x, y, z)-F(\infty, \infty, \infty)$

where the origin of the coordinate system is chosen as shown in Fig. 1 and $F(x, y, z)$ is the free energy of solvent in the case where the center of solute 1 is at position $(x, y, z)$. The entropic and energetic components of $\Phi\left(\Phi_{\mathrm{S}}\right.$ and $\Phi_{\mathrm{E}}$, respectively) are obtained from

$\Phi_{\mathrm{S}}=-(\partial \Phi / \partial T)_{\mathrm{V}}=-\{\Phi(T+\delta T)-\Phi(T-\delta T)\} /(2 \delta T), \delta T=5 \mathrm{~K}, \Phi_{\mathrm{E}}=\Phi+T \Phi_{\mathrm{S}}$.

We are particularly interested in $\Phi, \Phi_{\mathrm{S}}$ and $\Phi_{\mathrm{E}}$ within the vessel cavity which are largely influenced by the solvent structure within it.

\section{Results and Discussion}

The spatial distribution of $\Phi /\left(k_{\mathrm{B}} T\right)$ on the cross section $z=0$ and its components, $\Phi_{\mathrm{E}} /\left(k_{\mathrm{B}} T\right)$ and $-\Phi_{\mathrm{S}} / k_{\mathrm{B}}$, are shown in Fig. 2 where $\xi_{1} /\left(k_{\mathrm{B}} T\right)=1.5$ for $T=298 \mathrm{~K}$ and $d_{\mathrm{B}}=5 d_{\mathrm{S}}$. In Fig. 2(a), a domain within which the solute is highly stabilized appears around the $x$-axis. It is difficult for the solute to overcome a free-energy barrier scaled by $k_{\mathrm{B}} T$ well exceeding 1. As explained in the figure caption, the solute is most likely to be inserted into the vessel through the route indicated by the white dotted arrow and constrained within the small space around the position indicated by "-5.9" (i.e., almost in the center of the vessel cavity: This is consistent with an experimental observation [21]). There is symmetry along the $y$-axis. The route given by the white dotted arrow is just an example one. Of course, the route that is symmetrical about the $y$-axis is also equally probable. We then compare $\Phi /\left(k_{\mathrm{B}} T\right), \Phi_{\mathrm{E}} /\left(k_{\mathrm{B}} T\right)$, and $-\Phi_{\mathrm{S}} / k_{\mathrm{B}}$ within the domain around the $x$-axis. Insertion and constraint of the solute is achieved by $-\Phi_{\mathrm{S}} / k_{\mathrm{B}}$. This is 
consistent with the result of our recent study [15] where the solvent is modeled as hard spheres and no attractive potentials are considered.

In Fig. 3(a), the parameter setting is changed to " $\xi_{1} /\left(k_{\mathrm{B}} T\right)=0.0$ and $d_{\mathrm{B}}=5 d_{\mathrm{S}}$ ". As observed in Fig. 3(a), $\Phi_{\mathrm{E}} /\left(k_{\mathrm{B}} T\right)$ acts for insertion when the solute is solvophobic. In view of the difference between Fig. 2(b) and Fig. 3(a) and judging from the results of more calculations performed, we conclude that $\Phi_{\mathrm{E}} /\left(k_{\mathrm{B}} T\right)$ acts for release when the solute is solvophilic. This is because a solvophilic solute is preferentially solvated in the bulk solvent. By contrast, a solvophobic solute tends to be excluded from the bulk and inserted into the vessel cavity. In Fig. 3(b), the parameter setting is changed to " $\xi_{1} /\left(k_{\mathrm{B}} T\right)=1.5$ for $T=298 \mathrm{~K}$ and $d_{\mathrm{B}}=3 d_{\mathrm{S}}$ ". As observed in Fig. 2(c) and Fig. 3(b), a decrease in the solute size $d_{\mathrm{B}}$ gives only a smaller EV for solvent molecules, leading to weaker entropic force and smaller amplitudes of $-\Phi_{\mathrm{S}} / k_{\mathrm{B}}$ (i.e., smaller $\left|-\Phi_{\mathrm{S}} / k_{\mathrm{B}}\right|$ ). As a consequence, the insertion power induced by $-\Phi_{\mathrm{S}} / k_{\mathrm{B}}$ becomes weaker as $d_{\mathrm{B}}$ decreases. Another finding is that the qualitative aspects of $-\Phi_{\mathrm{S}} / k_{\mathrm{B}}$ are less sensitive to the parameter setting than $\Phi_{\mathrm{E}} /\left(k_{\mathrm{B}} T\right)$.

The solute size needs to be made smaller for releasing the solute, which has already been inserted into the vessel and is constrained inside it, to the outside. The EV then decreases, leading to a reduced insertion power induced by $-\Phi_{\mathrm{S}} / k_{\mathrm{B}}$. At the same time, when the solute solvophilicity is sufficiently increased, $\Phi_{\mathrm{E}} /\left(k_{\mathrm{B}} T\right)$ acts for release. If $\Phi_{\mathrm{E}} /\left(k_{\mathrm{B}} T\right)$ is dominant, the solute is released. This argument is demonstrated in Fig. 4 where $\xi_{1} /\left(k_{\mathrm{B}} T\right)=3.0$ for $T=298 \mathrm{~K}$ and $d_{\mathrm{B}}=3 d_{\mathrm{S}}$. As observed in Fig. $4(\mathrm{a})$, the solute is most likely to be released from the vessel cavity to the outside through the route indicated by the white dotted arrow. The release is made possible by $\Phi_{\mathrm{E}} /\left(k_{\mathrm{B}} T\right)$. In example (II), since the unfolded protein generates a large $\mathrm{EV}$, it feels $-\Phi_{\mathrm{S}} / k_{\mathrm{B}}$ which strongly drives its insertion into GroEL. Its overall solvophilicity is low, and $\Phi_{\mathrm{E}} /\left(k_{\mathrm{B}} T\right)$ felt by it promotes only weak release or insertion. Consequently, it is inserted. The folded protein, by contrast, possesses a smaller EV and much higher overall solvophilicity, and $-\Phi_{\mathrm{S}} / k_{\mathrm{B}}$ drives its insertion less strongly while $\Phi_{\mathrm{E}} /\left(k_{\mathrm{B}} T\right)$ powerfully acts for release: It is released.

The cycle comprising the binding of ATP to GroEL, hydrolysis of ATP into Pi and ADP, and release of $\mathrm{Pi}$ and ADP is in full play [3,4]. The ATP binding induces the binding of GroES to GroEL and causes a structural change of GroEL. GroES works as a lid. Without the lid binding, the inner surface of GroEL is rather hydrophobic [3]. This is consistent with the rather small value of $\xi_{2}$ in our parameter setting. Upon the lid binding, the inner surface becomes somewhat hydrophilic. We have performed 
additional calculations using a simple lid model and the vessel whose inner surface is solvophilic. We have verified the following: With the lid binding, only the PMF-values in the vicinity of inner surfaces of the lid and GroEL undergo significant changes; and an unfolded protein is repelled from the lid, still inserted into a small space almost in the center of the GroEL cavity, and constrained within it. This behavior is in accord with the experimental observation [4]. After the folding is finished, the release of Pi and ADP occurs. This is followed by the unbinding of the lid, leading to the PMF looking like that in Fig. 4(a). The folded protein is then released to the outside.

\section{Concluding Remarks}

We have developed a theoretical model which consistently elucidates both of insertion and release of a large solute into and from a biopolymer complex. Insertion is entropically driven. The switch from insertion to release is achieved by reducing the EV generated by the solute for solvent molecules and by increasing the solute solvophilicity. The reduction weakens the insertion power induced by the entropic force, and the increase promotes preferential solvation of the solute in the bulk solvent. The latter effect, which dominates in the release process, is not entropic but energetic in origin. Insertion of an unfolded protein into GroEL followed by release of the folded protein can be understood through this mechanism. A protein molecule is heterogeneous in the sense that hydrophobic and hydrophilic groups are almost randomly distributed within it. Even after its folding is completed, the increase in its surface hydrophilicity is not very large. Therefore, the reduction in its excluded volume is also required for the release. Specific, chemical characteristics of each protein are not very important, which is in accord with the experimental evidence [3] that a variety of proteins are inserted and released. Further, we have revealed a new aspect of high function of the water confined on the scale of a nanometer. (Explication of leading roles of water in biological functions for sustaining life is an imperative subject $[13,14]$.) As the next step, we intend to investigate effects of factor (A) in examples (I) and (II) by accounting for the polyatomic structure of the vessel. 


\section{Acknowledgment}

This work was supported by Grants-in-Aid for Scientific Research on Innovative Areas (No. 20118004) and that on (B) (No. 22300100) from the Ministry of Education, Culture, Sports, Science and Technology of Japan, by the Grand Challenges in Next-Generation Integrated Nanoscience, MEXT, Japan, and by Kyoto University Global Center of Excellence of Energy Science.

\section{References}

[1] K. Hollenstein, R. J. P. Dawson, and K. P. Locher, Curr. Opin. Struct. Biol. 17 (2007) 412.

[2] A. Ward, C. L. Reyes, J. Yu, C. B. Roth, and G. Chang, Proc. Natl. Acad. Sci. USA 104 (2007) 19005.

[3] A. L. Horwich, W. A. Fenton, E. Chapman, and G. W. Farr, Annu. Rev. Cell. Dev. Biol. 23 (2007) 115.

[4] R. Kanno, A. Koike-Takeshita, K. Yokoyama, H. Taguchi, and K. Mitsuoka, Structure 17 (2009) 287.

[5] A. S. Oliveira, A. M. Baptista, and C. M. Soares, J. Phys. Chem. B 114 (2010) 5486.

[6] F. Takagi, N. Koga, and S. Takada, Proc. Natl. Acad. Sci. U.S.A. 100 (2003) 11367.

[7] J. L. England, D. Lucent, and V. S. Pande, J. Am. Chem. Soc. 130 (2008) 11838.

[8] M. Kinoshita, J. Chem. Phys. 116 (2002) 3493.

[9] R. Roth, R. van Roij, D. Andrienko, K. R. Mecke, and S. Dietrich, Phys. Rev. Lett. 89 (2002) 088301.

[10] Y. Harano and M. Kinoshita, Biophys. J. 89 (2005) 2701.

[11] M. Kinoshita, Chem. Eng. Sci. 61 (2006), 2150.

[12] P.-M. König, R. Roth, and S. Dietrich, Europhys. Lett. 84 (2008) 68006.

[13] M. Kinoshita, Front. Biosci. 14 (2009) 3419.

[14] M. Kinoshita, Int. J. Mol. Sci. 10 (2009) 1064.

[15] K. Amano and M. Kinoshita, Chem. Phys. Lett. 488 (2010) 1.

[16] K. Amano, T. Yoshidome, M. Iwaki, M. Suzuki, and M. Kinoshita, J. Chem. Phys. 133 (2010) 045103. 
[17] M. Ikeguchi and J. Doi, J. Chem. Phys. 103 (1995) 5011.

[18] M. Kinoshita, Mol. Phys. 94 (1998) 485.

[19] M. Kinoshita, Mol. Phys. 96 (1999) 71.

[20] M. Kinoshita, J. Chem. Phys. 128 (2008) 024507.

[21] H. Saibil and S. Wood, Curr. Opin. Struct. Biol. 3 (1993) 207. 


\section{Figure Captions}

Fig. 1. Large sphere and cross section of vessel considered $(z=0)$. The numbers given are scaled by $d_{\mathrm{S}}$. When the large-sphere diameter $d_{\mathrm{B}}$ is set at $5 d_{\mathrm{S}}$, for example, the center of the large sphere in contact with the bottom wall is at $(x, y)=(0,0)$.

Fig. 2. Spatial distribution of $\Phi /\left(k_{\mathrm{B}} T\right)$ (a), $\Phi_{\mathrm{E}} /\left(k_{\mathrm{B}} T\right)(\mathrm{b})$, and $-\Phi_{\mathrm{S}} / k_{\mathrm{B}}$ (c) on the cross section $z=0$ between the large sphere and the vessel: $\xi_{1} /\left(k_{\mathrm{B}} T\right)=1.5$ for $T=298 \mathrm{~K}$ and $d_{\mathrm{B}}=5 d_{\mathrm{S}}$. As the color approaches thick blue, they become lower, and as the color approaches thick red, they become higher ("max" and "min" represent the maximum and minimum values, respectively). The center of the large sphere cannot enter the domain drawn in white. The values at the positions indicated by the black broken arrows are also given. The numbers with the black solid arrows denote the free-energy barriers. In (a), the large sphere must overcome the barrier scaled by $k_{\mathrm{B}} T$ of $\sim 4$ to move from the position of " -5.9 " to that of " -7.3 ": It is constrained within the small space around the position indicated by " -5.9 ".

Fig. 3. (a) Spatial distribution of $\Phi_{\mathrm{E}} /\left(k_{\mathrm{B}} T\right)$ on the cross section $z=0$ between the large sphere and the vessel: $\xi_{1} /\left(k_{\mathrm{B}} T\right)=0.0$ and $d_{\mathrm{B}}=5 d_{\mathrm{S}}$. As the color approaches thick blue, they become lower, and as the color approaches thick red, they become higher ("max" and "min" represent the maximum and minimum values, respectively). The center of the large sphere cannot enter the domain drawn in white. The values at the positions indicated by the black broken arrows are also given. This figure is to be compared with Fig. 2(b). (b) Spatial distribution of $-\Phi_{\mathrm{S}} / k_{\mathrm{B}}$ on the cross section $z=0$ between the large sphere and the vessel: $\xi_{1} /\left(k_{\mathrm{B}} T\right)=1.5$ for $T=298 \mathrm{~K}$ and $d_{\mathrm{B}}=3 d_{\mathrm{S}}$. This figure is to be compared with Fig. 2(c).

Fig. 4. Spatial distribution of $\Phi /\left(k_{\mathrm{B}} T\right)$ (a), $\Phi_{\mathrm{E}} /\left(k_{\mathrm{B}} T\right)(\mathrm{b})$, and $-\Phi_{\mathrm{S}} / k_{\mathrm{B}}$ (c) on the cross section $z=0$ between the large sphere and the vessel: $\xi_{1} /\left(k_{\mathrm{B}} T\right)=3.0$ for $T=298 \mathrm{~K}$ and $d_{\mathrm{B}}=3 d_{\mathrm{S}}$. As the color approaches thick blue, they become lower, and as the color approaches thick red, they become higher ("max" and "min" represent the maximum and minimum values, respectively). The center of the large sphere cannot enter the domain drawn in white. The values at the positions indicated by the black broken arrows are also given. 


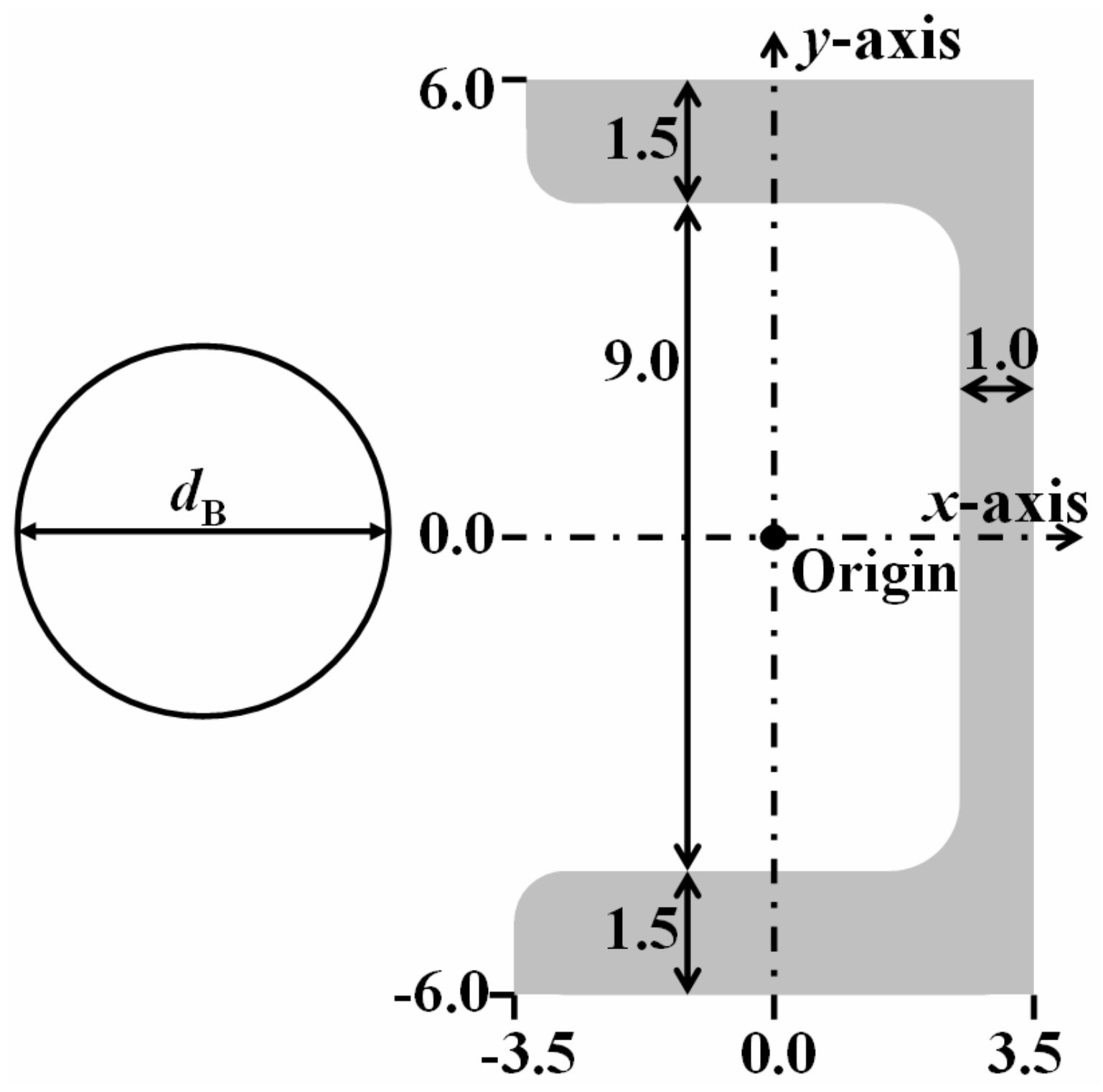

Fig. 1 

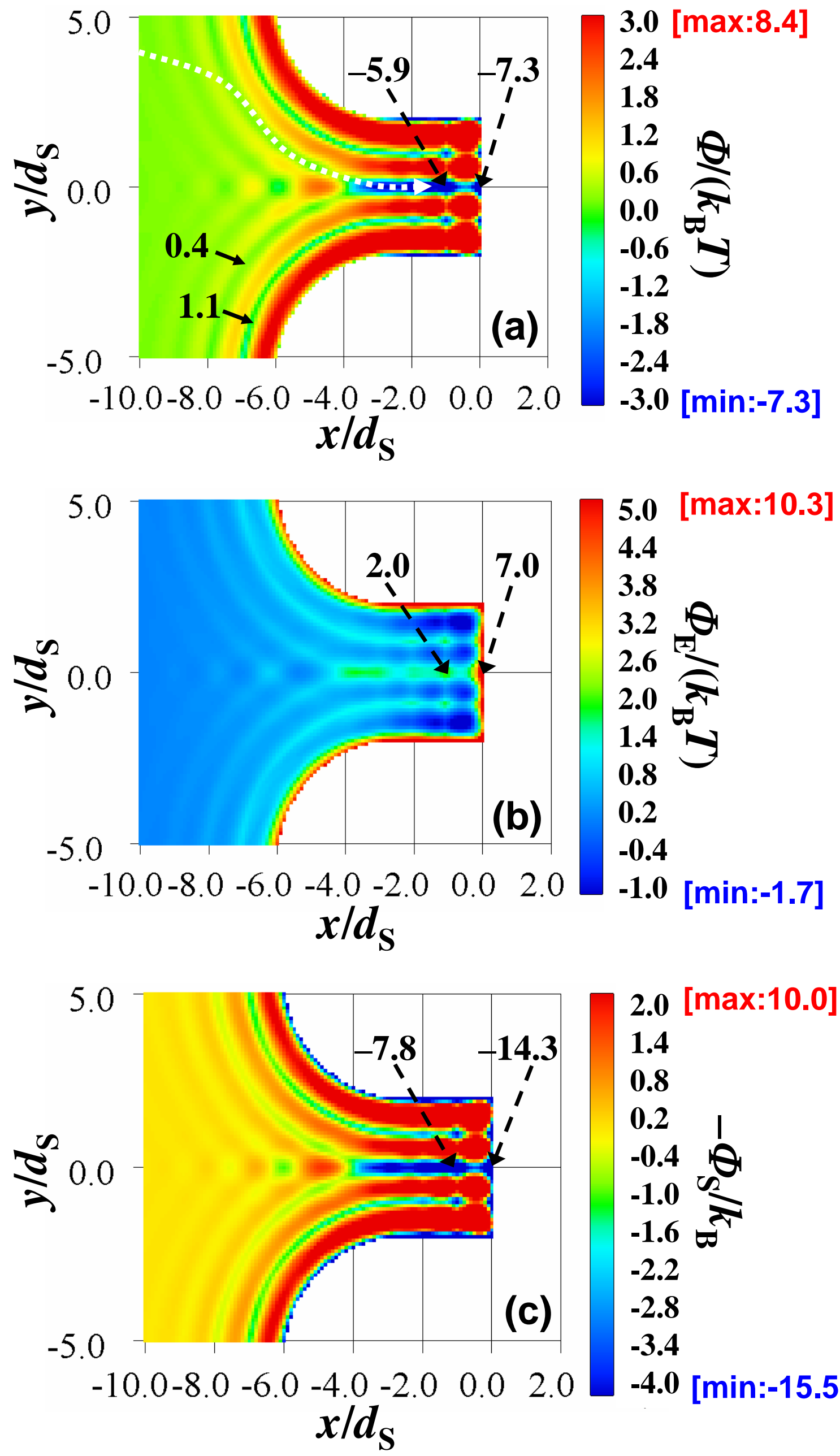

2.0 [max:10.0]

1.4

0.8

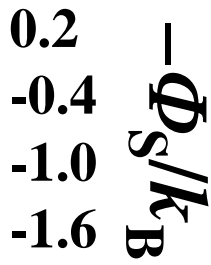

$-2.2$

$-2.8$

$-3.4$

-4.0 [min:-15.5] $x / d_{\mathrm{S}}$

Fig. 2 

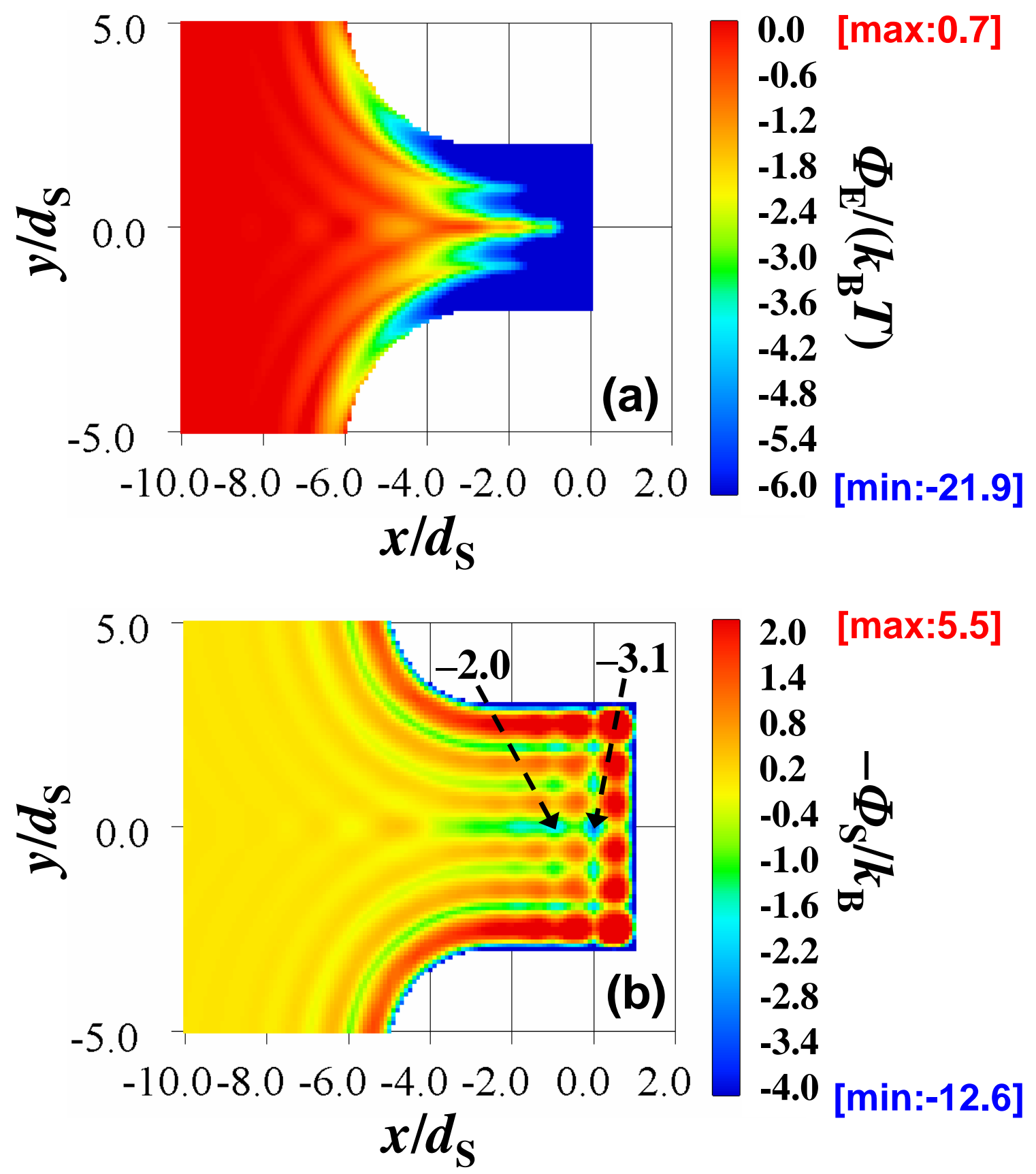

Fig. 3 

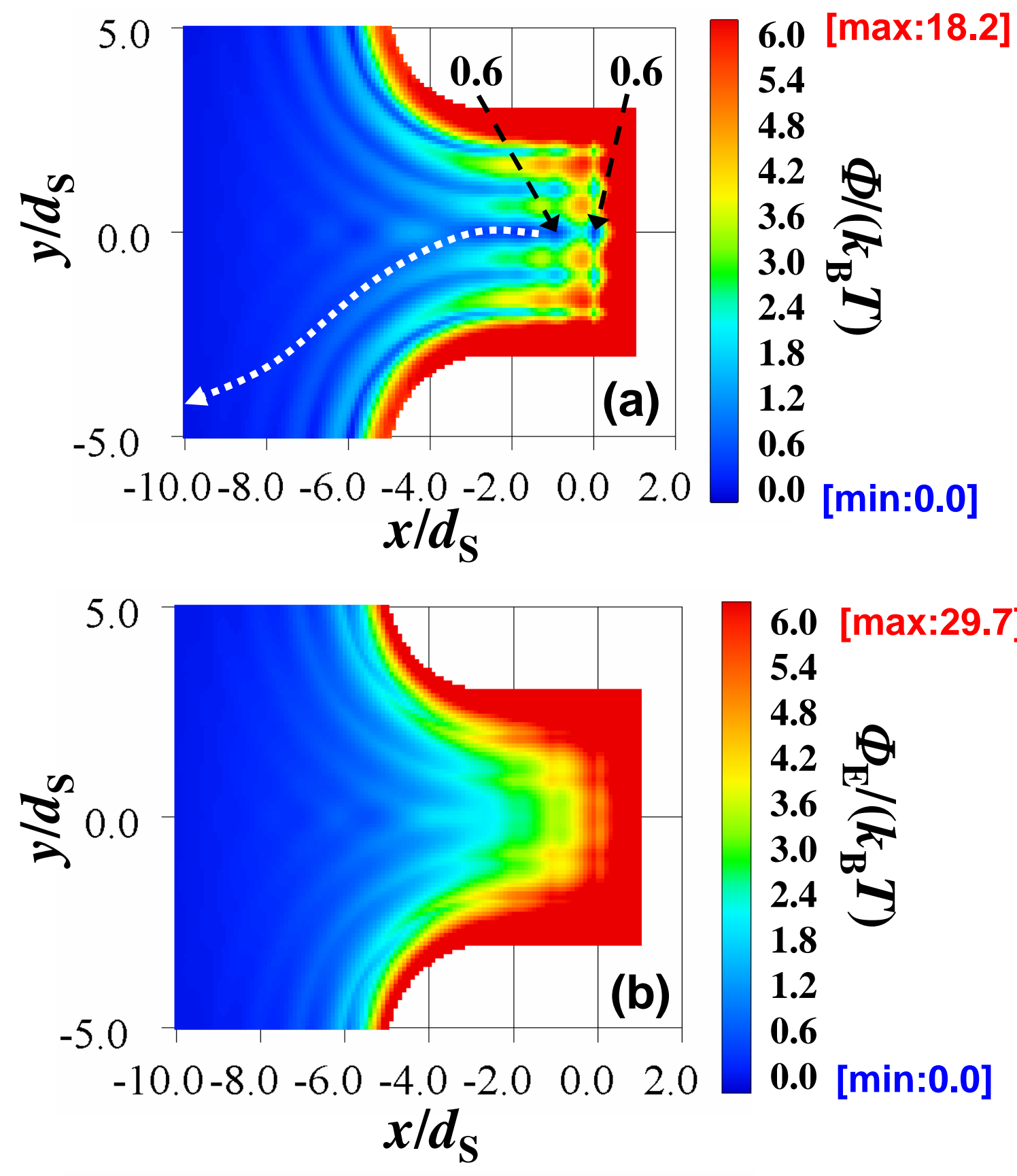

6.0 [max:29.7] 5.4 4.8

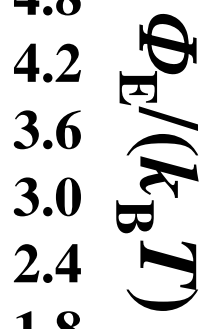
1.8 1.2 0.6 0.0 [min:0.0] $x / d_{\mathrm{S}}$

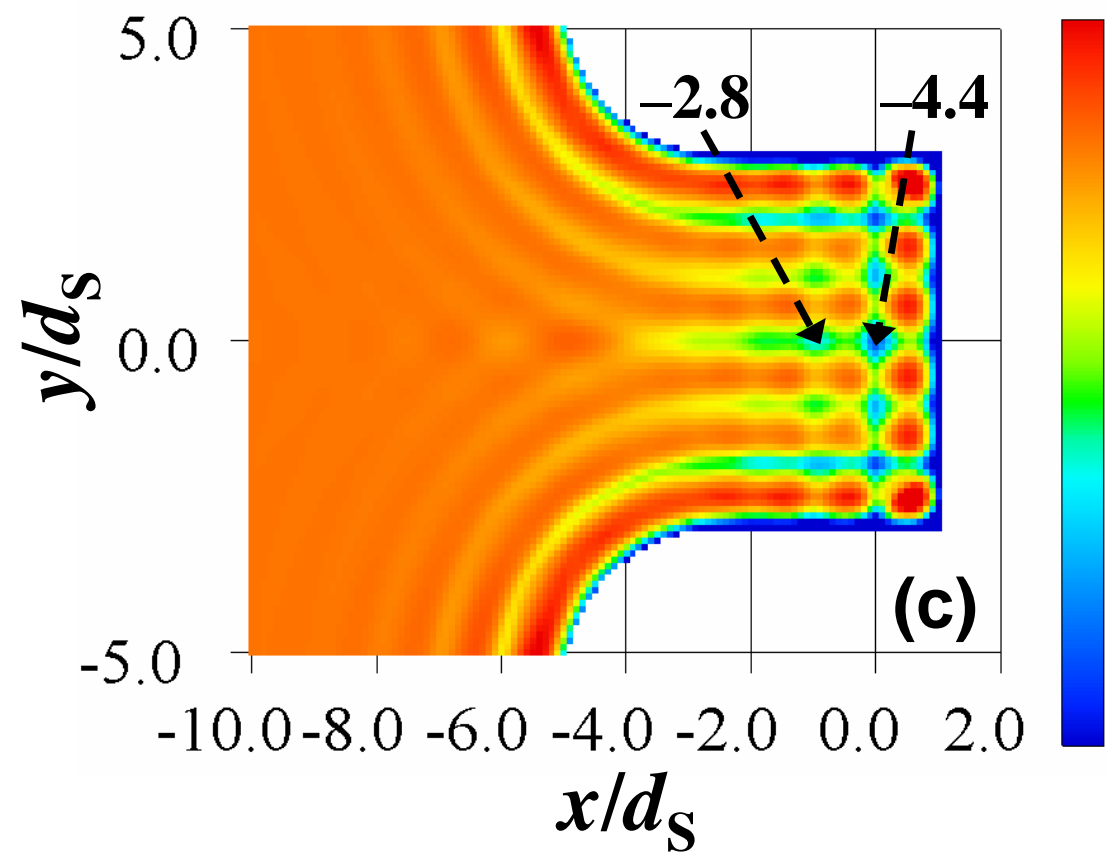

1.0 [max:2.0] 0.4 $-0.2$ $-0.8$

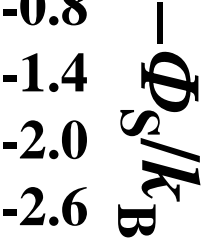
$-3.2$

$-3.8$ $-4.4$ -5.0 [min:-15.6] $x / d_{\mathrm{S}}$

Fig. 4 